\title{
Important considerations when providing mental health first aid to Iraqi refugees in Australia: a Delphi study
}

\author{
Maria Gabriela Uribe Guajardo ${ }^{*} \mathbb{D}$, Shameran Slewa-Younan' ${ }^{1}$ Yvonne Santalucia² and Anthony Francis Jorm ${ }^{3}$
}

\begin{abstract}
Background: Refugees are one of the most vulnerable groups in Australian society, presenting high levels of exposure to traumatic events and consequently high levels of severe psychological distress. While there is a need for professional help, only a small percentage will receive appropriate care for their mental health concerns. This study aimed to determine cultural considerations required when providing mental health first aid to Iraqi refugees experiencing mental health problems or crises.

Method: Using a Delphi method, 16 experts were presented with statements about possible culturally-appropriate first aid actions via questionnaires and were encouraged to suggest additional actions not covered by the questionnaire content. Statements were accepted for inclusion in a guideline if they were endorsed by $\geq 90 \%$ of panellists as 'Essential' or'Important'.

Results: From a total of 65 statements, 38 were endorsed ( 17 for cultural awareness, 12 for cross-cultural communication, 7 for stigma associated with mental health problems, and 2 for barriers to seeking professional help).

Conclusion: Experts were able to reach consensus about how to provide culturally-appropriate first aid for mental health problems to Iraqi refugees, demonstrating the suitability of this methodology in developing cultural considerations guidelines. This specific refugee study provided potentially valuable cultural knowledge required to better equip members of the Australian public on how to respond to and assist Iraqi refugees experiencing mental health problems or crises.
\end{abstract}

Keywords: Mental health first aid, Iraqi refugees, Mental health problems, Delphi method

\section{Background}

Australia is a multicultural country with almost a quarter $(24.6 \%)$ of its population born overseas and $43.1 \%$ of people with at least one overseas-born parent [1]. Over the period 2003-2004 to 2012-2013, Australia resettled 146,321 refugees [2]. Most recently, the Australian Humanitarian Program 2013-2014 granted 13,768 visas to onshore and offshore visa applicants [3]. Currently, Iraq is one of the top source countries for refugee applications to Australia [3]. Further, in 2015, and in response to the conflicts in Syria and Iraq, the Australian

\footnotetext{
*Correspondence: m.uribe@westernsydney.edu.au

${ }^{1}$ Centre for Health Research, School of Medicine, Western Sydney University, Sydney, Australia

Full list of author information is available at the end of the article
}

Government announced an extra 12,000 humanitarian places available. People in these 12,000 places will be granted a permanent visa [4].

The presence of mental disorders amongst refugees has been well identified and explored for over 30 years [5-7]. Recent evidence has shown that prevalence of mental disorders and general psychological distress are both high in resettled Iraqi refugees $[8,9]$, with some experiencing severe psychological distress beyond the early resettlement period, even years after first arrival [9]. It has also been documented that while there is great need for specialised health care, only a small percentage of refugees will seek professional help [10], waiting until problems become critical before reaching specialist psychiatric and mental health services [11]. There may be 
several reasons why resettled Iraqi refugees do not seek help. Some of these may include stigma associated with mental health problems [12], inability to recognise mental disorders [13], lack of knowledge of treatment and intervention available in the host country [13] and migration challenges (e.g. cultural shock, lack of knowledge of the new country and its system, financial constraints, low language proficiency) [14].

Mental health first aid is 'the help offered to a person developing a mental health problem, experiencing a worsening of an existing mental health problem or in a mental health crisis. The first aid is given until appropriate professional help is received or until the crisis resolves' ( $\mathrm{p}$. 12) [21]. A Mental Health First Aid (MHFA) training program was introduced in Australia in 2001 with the goal of improving recognition of mental disorders, reducing stigma and promoting appropriate help-seeking, selfcare and support from others in the community [15]. The Standard MHFA course is delivered over 12-h of training and teaches the application of a first aid action-plan to mental health problems [21]. It has since been evaluated in multiple studies in Australia and other countries, demonstrating its acceptability and effectiveness [16]. Remarkably, since 2001 MHFA has been disseminated rapidly and gained recognition, with over 20 countries adopting the MHFA course and adapting it to their specific needs [17-20].

To increase the evidence base of the MHFA program, a series of Delphi studies have been conducted to develop expert consensus guidelines on what constitutes appropriate mental health first aid for a range of mental health problems or crises, such as depression, anxiety, panic attacks, traumatic events, and suicidal behaviours [21]. This type of consensus study has been used widely in mental health research [22] and has also served in the development of additional guidelines on provision of culturally-specific mental health first aid for minorities in Australia [23, 24]. Minas and Jorm argue that 'The development of a locally relevant evidence base is a valuable approach where other evidence is unavailable' and that expert consensus methods are a way of achieving this ( $\mathrm{p}$. 2) [25]. The current study sought to develop guidelines on important additional considerations when providing mental health first aid to Iraqi refugees.

\section{Cultural factors and mental health in refugees}

When assessing mental health needs of refugees, it is crucial to consider the complexity of the underlying phenomena and impact of various cultural perspectives, including Western approaches, in guiding understanding of mental illness. Over the past two decades, there has been a growing belief that the Western diagnostic and treatment systems for mental disorders may be insufficient or even in some cases inappropriate in crosscultural settings [26]. Since then, it has become evident that gaining an appreciation of individuals' understanding of mental health, their knowledge of treatments and willingness to access care for mental health concerns is essential when designing mental health services for the care of underrepresented minority groups (e.g. refugees or migrants) [27].

As such, in recent years, targeted research has emerged with the aim of exploring levels of mental health literacy amongst refugee groups in Australia [13, 28, 29]. These studies have reported lower recognition of mental health problems and differing beliefs regarding treatment approaches in refugee groups when compared to the Australian public [30]. Conclusions from these studies have highlighted the need for specific mental health education for refugees and those who care for them that embraces both Western mental health service approaches and traditional cultural and religious practices [13, 29].

Such culturally-responsive approaches have been used to help develop general health clinics [31], as well as other initiatives where asylum seekers and refugees have been trained to provide psycho-education and psychosocial support to fellow groups of refugees and asylum seekers [32].

This current study is expected to build cultural capacity at an Australian community level, by using a validated consensus method, which is deemed to be essential for effective cross-cultural interventions [23, 24, 33].

\section{The need for guidelines when assisting Iraqi refugees}

Refugees have an elevated risk of mental disorders in the resettlement period as a consequence of significant personal disruption and experiences of torture, trauma, and loss that many have experienced [34], along with postdisplacement challenges such as poor English language ability, unemployment, housing shortage and low educational opportunities [35].

In order to overcame the barriers in seeking professional help, researchers and clinicians are encouraged to develop alternative models of care to accommodate individuals' cultural and linguistic backgrounds, including meanings of emotion, suffering, trauma and support in their original and host cultural contexts [11]. However, much more needs to be done to enhance mental health service utilisation amongst people from refugee backgrounds. One way forward is to develop community-based culturally-appropriate interventions which can tangibly benefit refugees by seeking to reduce their psychological distress at different points of their resettlement journey.

As such, this study sought to explore what culturallyappropriate mental health first aid strategies have been proposed for assisting Iraqi refugees in mental health 
crises or developing mental health problems and which of these are considered by experts in the field to be the most appropriate strategies for people who are assisting this group. The study used the Delphi consensus method by identifying and recruiting experts to rate the importance of action statements on how to provide culturallyappropriate mental health first aid to Iraqi refugees.

\section{Methods}

\section{The Delphi method}

First developed to examine the impact of technology on warfare in 1950s, it has been defined as a type of consensus method, often using a non-face-to-face technique, for structuring a group communication process, allowing a group of individuals to deal with a complex problem $[36,37]$. It has been proven to be a feasible method when developing culturally-adapted interventions in mental health for minority and diverse groups $[38,39]$. This method involves questionnaires being sent out online to a group of experts, where responses are anonymous. The Delphi technique involves a number of iterations before consensus is reached. Feedback from the expert group as a whole is given in order to assist panellists to assess their ratings against the group feedback.

The development of these guidelines involved 8 steps: (1) framing a research question, (2) formation of the panel (3) determining the expert panel size, (4) constructing the questionnaire, (5) information provided to panel members to aid their judgements, (6) administering the questionnaire, (7) analysing rounds and providing feedback to the panel and (8) reporting results.

\section{Research question}

This study aimed to respond to the following questions: (1) what culturally appropriate mental health first aid strategies have been proposed for assisting Iraqi refugees in mental health crises or in the development of mental health problems? and (2) which of these are considered by experts in the field to be the most appropriate strategies for assisting this group?

\section{Formation of the panel}

The expert panel was composed of professionals who meet the following selection criteria: qualified as a psychologist, social worker, psychiatrist, general practitioner or mental health professional; and have worked in refugee mental health part-time or full-time for at least 4 years and have experience working with Iraqi refugees. Potential participants were considered to have sufficient expertise if they have authored material in Iraqi refugee mental health and/or were known as experienced professionals through different networks, such refugee health networks in Australia, professional associations or community involvement.

Potential participants were identified and selected to participate in this project through their involvement with professional colleges and associations, universities and research centres, refugee health networks and refugee health services in New South Wales, South Australia, Australian Capital Territory and Victoria, Australia. Expert panellists were contacted via multiple sources. The principal researcher (MGU) advertised the study in various professional associations such as The Australian Psychological Society, The Australian Association of Social Workers, and The Royal Australian and New Zealand College of Psychiatrists. In addition, an email invitation was circulated to those involved with key government and non-government refugee health organisations and university centres. In the invitation to participate, professionals were asked to share the study invitation with colleagues who they felt would be appropriate panel members. This research was granted human research ethics approval by the Western Sydney University Human Research Ethics Committee (H11054). Consent and participation information sheet forms were sent by email or post to participants. Signed consent forms were collected by the principal researcher (MGU) before the study commencement. Participants were reimbursed for their time with a book voucher of $\$ 50$.

\section{Determining expert panel size}

A panel size of 23 has been found to yield stable results in a simulation study [40]. Our aim was for a minimum of 30 members in the panel, in order to allow for drop-outs. However, a total of 16 experts were recruited.

\section{Constructing the questionnaire}

A systematic literature search was carried out to identify available information about how to provide culturallyappropriate mental health first aid to Iraqi refugees experiencing mental health problems or crises. This search focused in three main sources. A comprehensive search of key terms was carried out using Google search engines (http://www.google.com.ca, http://www.google.com.uk, http://www.google.com.au, http://www.google.com). The searches included various combinations of search terms, for example, 'mental health in Iraqi refugees' AND 'early mental health intervention for Iraqi refugees' AND 'first aid for Iraqi refugees' AND 'cultural considerations for Iraqi refugee mental health'. Lists of the first 50 websites for each set of terms were reviewed. Any links of interest appearing in the websites were followed, as appropriate. A second strategy was academic journal search using Medline, PsycINFO, CINAHL, PILOTS, Scopus and Cochrane databases to identify relevant published 
articles. A third strategy was searching for any printed books available in Australian-wide libraries using Trove Australia, Libraries Australia and Australian Libraries Getaway (ALG) search catalogues. Information from relevant mental health and refugee websites such as Transcultural Mental Health, Australian Psychological Society, Mental Health Australia and United Nations High Commissioner for Refugees (UNHCR) were also reviewed.

In order to develop the first round questionnaire, information obtained from the systematic literature review was divided into common sections or categories and were written as strategies or actions a first aider should follow. For example, in a report entitled 'The Mental and Physical Health of Recent Iraqi refugees' it was stated that 'talking about mental health openly is often stigmatised by Iraqis'. This statement was included in the first round questionnaire under the category 'stigma associated with mental health problems' as a first aid action as follows: 'The first aider should be aware that talking about mental health problems openly is often stigmatised by the Iraqi community'.

The process of phrasing and drafting statements into a questionnaire format involved a team of authors, who were experts in the Delphi method (AJ) or in transcultural mental health (MGU, SSY, YS). The team drafted the actions and attempted to remain as close to the original literature as possible. Statements were only modified in order to ensure format consistency or content comprehension. Several meetings were held to discuss the items before the final round 1 questionnaire was ready to be sent. In total, three questionnaires were created and presented to experts in a 3-round format.

\section{Information provided to experts to aid their responses}

Questionnaires were accompanied by definitions of key concepts that were thought to be relevant for experts when making their ratings. These definitions included the role of a 'first aider', the 'person' defined as an 'Iraqi refugee' and 'people' defined as 'Iraqi people in general', as well as definitions of 'mental Illness', 'cross-cultural communication' and 'cultural awareness'.

\section{Administering the questionnaire and analysing rounds}

The majority of participants chose to complete the questionnaire (round 1) online and a link was sent hosted by http://www.SurveyMonkey.com to their nominated email addresses. A small number of experts preferred to complete a hard copy questionnaire. Experts responded to each statement by rating how essential the first aid action statements were to the development of guidelines on how to provide mental health first aid to Iraqi refugees experiencing mental health problems or crises.
The questionnaire involved a 5-point Likert scale composed of 'Essential', 'Important', 'Depends/don't know', 'Unimportant' and 'Should not be included' as response options.

Once the panel rating was completed, actions were categorized based of level of consensus following the procedure used in a previous Delphi study [23]:

1. If between 90 and $100 \%$ of panel members rated a statement as either 'Essential' or 'Important', the statement was endorsed as a guideline.

2. If between 80 and $89 \%$ of panel members rated a statement as either 'Essential' or 'Important', then the statement was entered into a second questionnaire to be rerated (second round).

3. If neither of the above conditions were met then the statement was excluded from the guidelines.

In round 1 , in addition to rating statements, participants were encouraged to provide feedback on any ambiguity in the statements presented and suggest any new first aid strategies that were not included in the content of the questionnaire. Submitted comments were drafted into statements and then presented to the working group, who tried to ensure comprehensibility and consistency. Those statements that were assessed by the working group to be original were included as new actions in a second round questionnaire for experts to rate.

Once categorisation was completed, participants were sent a report that included endorsed, rejected and new statements to be rated along with those statements that needed to be re-rated in the next round questionnaire. The statements to be re-rated were presented with the group percentages for each possible rating, and also with the individual's response for the expert to compare their own rating to the group response. By providing this report to the experts, researchers aimed to aid participants' re-rating.

In round 2, the same criteria were followed for endorsing, excluding and re-rating statements. However, those statements that failed to meet the criteria for endorsement in the second round were then excluded from the guidelines. Only those new statements that were introduced in round 2, and afterward fell into the re-rate category, were in a third and final round.

\section{Results}

Sixteen panel members were recruited ( 7 male, 9 female, mean age $=44.37$; $\mathrm{SD}=12.46)$ with $100 \%$ retention across the three rounds. The majority of recruited participants were based in Australia, with only one expert based in the Netherlands. Eleven participants were from 
New South Wales, three from South Australia and one from Australian Capital Territory.

The majority worked in the private sector and years of experience ranged from 4 to 40 (mean $=16.55$; $\mathrm{SD}=11.44$ ) with more than $40 \%$ having 16 or more years of experience.

Participants' professions varied and included: consultant psychiatrists $(n=2)$, psychologists $(n=4)$, general practitioners $(\mathrm{n}=4)$, medical director $(\mathrm{n}=1)$, case managers/social workers $(n=3)$, mental health project officer $(\mathrm{n}=1)$ and occupational therapist $(\mathrm{n}=1)$. It is important to note that some of the participants held both professional and academic appointments $(\mathrm{n}=3)$. Most of participants had attained a post-graduate degree $(\mathrm{n}=11)$.

\section{Endorsed statements}

Of the 65 statements presented to the experts, including those statements that were drafted from experts' feedback, 38 were endorsed as either essential or important to the development of guidelines on providing mental health first aid to Iraqi refugees (Fig. 1). A list of all endorsed statements can be found in Additional file 1. Endorsed items were categorised in four main topics as follows: cultural awareness, cross-cultural communication, stigma associated with mental health problems and barriers to seeking professional help (Table 1). The vast majority of endorsed statements fitted within the cultural awareness category due to the relatively larger literature in this area compared to the other categories. In terms of round inclusion, the majority of the statements were endorsed in round $1(\mathrm{n}=24)$, followed by round 2 $(n=14)$. There were no statements endorsed in round 3.

\section{Rejected statements}

A total of 27 statements were excluded from the guidelines as it failed to reach the $90 \%$ level as 'Important' or 'Essential' (Fig. 1). A list of rejected items can be found in Additional file 2. The majority of rejected statements were categorised under 'Cultural awareness', consistent with the pattern reported for endorsed statements (Table 1). Again, the majority of the statements were rejected in round 1.

\section{New statements}

In round 1, researchers encouraged panel members to provide feedback or any strategies they thought would be useful when providing mental health first aid to Iraqi refugees. A total of 11 new strategies based on the panel's feedback were incorporated in round 2 (Fig. 1). Six statements were endorsed as either essential or important by the panel. These statements were endorsed in round 2, with no new strategies endorsed in round 3.

\section{Discussion}

The current study sought to explore what experts in refugee mental health thought would be culturally-appropriate mental health first aid strategies for assisting Iraqi refugees in mental health crises or developing mental health problems. Experts were able to reach consensus on the importance of a set of culturally-specific guidelines on how to provide mental health first aid to Iraqi refugees with high levels of distress.

The first category that emerged was 'cultural awareness.' Research has demonstrated that understanding cultural norms, values and beliefs of a person from a different cultural background is essential when trying to help individuals in cross-cultural contexts [23, 24, 34]. Thus, this category represents the importance of cultural awareness when providing mental health first aid to Iraqi refugees. This awareness can aid the identification of presenting problems (mental health symptoms) and provision of appropriate assistance, going beyond standard mental health classifications. Specifically, members of the public who wish to assist resettled Iraqi refugees should be aware of wars and mass persecution that Iraq has endured for almost three decades, including internal and overseas displacement of its population. It is also recommended that aiders should have awareness of the diversity of ethnicities and religions in Iraq and among those who have being granted permanent resettlement in Australia. Further, aiders must also understand the impact of culture on attitudes, behaviours and beliefs associated with mental health problems and mental health risk factors in the Iraqi community. These strategies reflect the importance of complexity of Iraq's cultural, ethnic and religious homogeneity and how such differences can play a role when assisting individuals from Iraq.

It also became evident, as noted previously [23, 24, 34], that applying 'cross-cultural communication' strategies while communicating with individuals from a different culture is essential. According to the results of this study, aiders should be encouraged to communicate in a culturally sensitive and respectful way, being mindful of the limited English skills refugees may have, which can increase the potential for misunderstandings. There is no doubt that communicating with individuals with limited English skills or from culturally diverse backgrounds can be challenging, and that is why aiders are encouraged to promote the use of a professional interpreter when one is needed. Aiders should be aware of the environment and surroundings when trying to help refugees, avoiding places that might trigger traumatic memories of when they fled home, including their refugee journey (internally displaced or displaced in neighbouring countries) before arriving to Australia. Observing these 


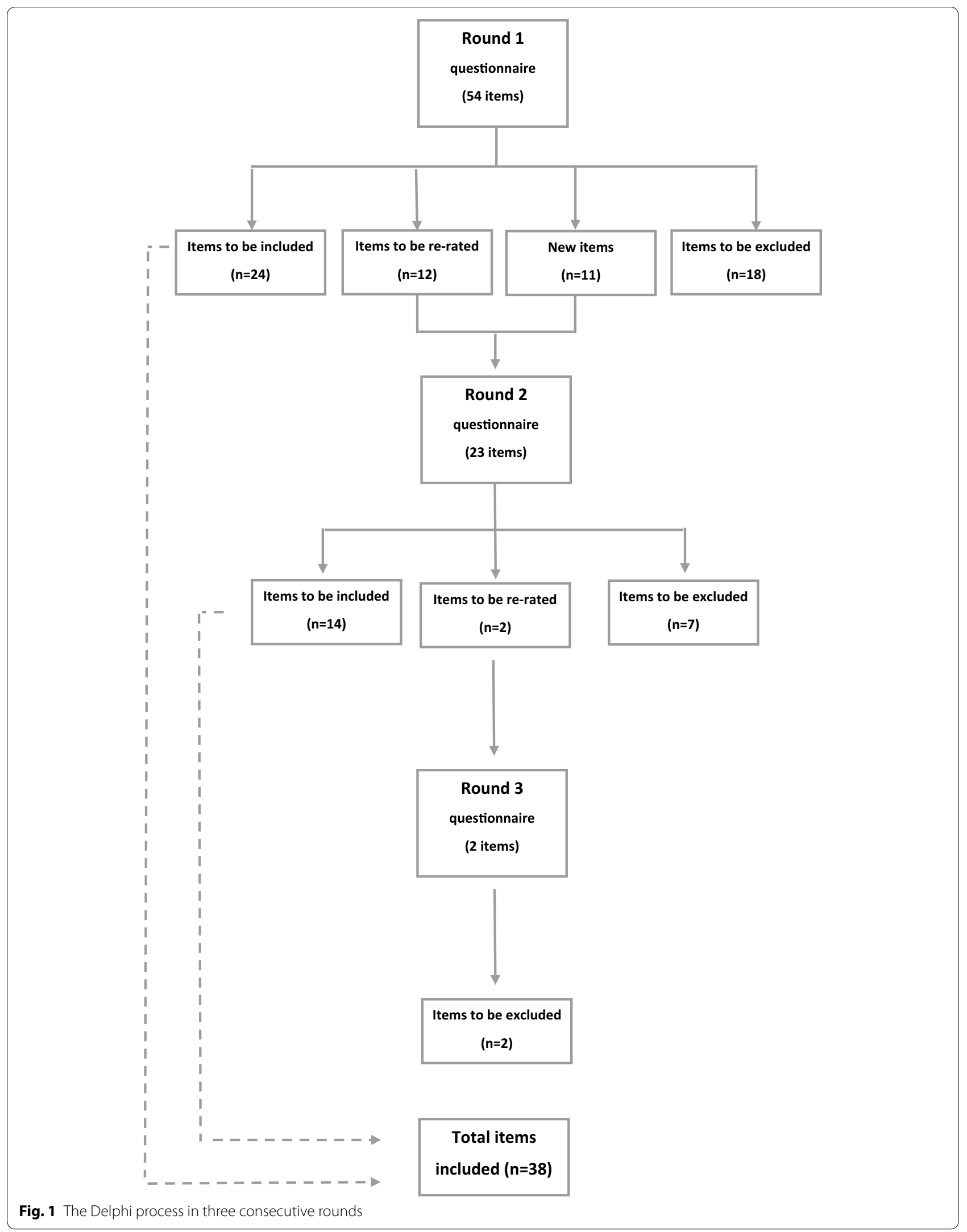


Table 1 Summary of endorsed and rejected statements by round and category

\begin{tabular}{|c|c|c|c|c|c|c|c|c|}
\hline \multirow[t]{3}{*}{ Round } & \multicolumn{8}{|l|}{ Items } \\
\hline & \multicolumn{4}{|l|}{ Endorsed } & \multicolumn{4}{|l|}{ Rejected } \\
\hline & $\begin{array}{l}\text { Cultural aware- } \\
\text { ness }\end{array}$ & $\begin{array}{l}\text { Cross-cultural } \\
\text { Communication }\end{array}$ & Stigma & $\begin{array}{l}\text { Barriers to seek- } \\
\text { ing help }\end{array}$ & $\begin{array}{l}\text { Cultural aware- } \\
\text { ness }\end{array}$ & $\begin{array}{l}\text { Cross-cultural } \\
\text { communication }\end{array}$ & Stigma & $\begin{array}{l}\text { Barriers to seeking } \\
\text { help }\end{array}$ \\
\hline 1 & 11 & 9 & 3 & 1 & 6 & 3 & 3 & 6 \\
\hline 2 & 6 & 3 & 4 & 1 & 4 & - & 2 & 1 \\
\hline 3 & - & - & - & - & - & 1 & 1 & - \\
\hline
\end{tabular}

recommended strategies is essential for anyone wanting to offer effective initial help to someone from an Iraqi refugee background. By undertaking such strategies, communication is enhanced and potential misunderstandings are avoided, allowing first aiders to effectively approach, assist and assess for mental health crises, listen, give information and encourage professional help and other supports, as recommended in the action plan taught in MHFA courses [21].

A third category that emerged was 'stigma associated with mental health problems' in the Iraqi community. Negative attitudes towards mental health problems have been reported as one of the major reasons preventing people from seeking professional help in Australia [30]. In line with this evidence, stigma associated with mental health problems seems to be a rule in many countries and across different groups [41-43]. Specifically, Bolton [12] reported that the stigma associated with mental disorders in Iraq seems to be greater than in other parts of the world. According to Bolton, social distance and the stigma of mental illness extends to the patients' families, with feelings of shame as a common feature [12]. The current study identified that Iraqi refugees can be considered as 'crazy' by their community if they speak out about their mental health concerns. Aiders should be mindful that Iraqis may keep their mental health problems as secret and may not trust authorities such as health professionals, and that this can act as a barrier.

The last set of recommendations were categorised as 'barriers to seeking professional help'. Professional help-seeking is defined as the assistance from professionals who have a legitimate and recognised professional role in providing relevant advice, support and/or treatment. These include specialist and generalist health care providers [44]. Research has indicated that only a small number of refugees will seek professional help for mental health problems, with non-professional helpseeking being the preferred source of help for refugees during a mental health crisis [45]. Hence it is essential for members of the public wanting to assist Iraqi refugees to be aware that only a limited number of refugees will seek professional help. There is also a tendency for men not to seek help for fear of appearing weak when they are expected to be strong and support their families. In addition, traditional beliefs and causes of mental health problems may also prevent Iraqis from seeking professional help. It is important to understand the great disruption and the new identities that some refugees are forced to assume. First aiders needed to be mindful that Iraq is a very patriarchal society where traditional beliefs and gender roles are strongly adhered to and that such beliefs can impact professional help-seeking from individuals.

Limitations in this study must be noted. This study utilised a volunteer process and experts were recruited through their association with key non-government and government organisations. This method may have failed to capture experts not directly connected to such organisations, but who may have a more grass roots connection in assisting Iraqi refugees. In addition, the sample size of this study was relatively small with only 16 panel members recruited. This number, while smaller than the optimal panel size of 23 demonstrated to yield stable results in a simulation study [40], is likely to be a reflection of the relatively limited number of experts available on Iraqi mental health.

Future directions should include ways to engage and recruit mental health professionals in order to ensure a larger sample size, as well as engaging with carers and consumers from refugee backgrounds to be part of a multiple 'expert panels' study. However, this may impose a new challenge due to the difficulty of recruiting samples of individuals with a refugee background [46].

Strength of this research includes that this is the first study aiming to establish culturally-appropriate evidencebased actions to assist individuals from an Iraqi refugee background. By building on the highly-successful standard MHFA training, our guidelines will inform tailored education on how to help an Iraqi refugee presenting with common mental health problems such as depression and posttraumatic stress disorder among others. Additionally, while this study focussed on a specific refugee group, this method appears well suited in developing other cultural specific guidelines. Future directions will include the delivery 
and evaluation of this tailored educational program, which is currently underway and involves recruiting and training community-based workers assisting resettling Iraqi refugees in Western Sydney, Australia. It is anticipated that this tailored training will form the first line of a two-pronged approach seeking to improve the mental health outcomes of Iraqi refugees, with the second being mental promotion and educational strategies targeted towards the Iraqi refugees themselves.

\section{Conclusion}

In summary, our Delphi study is the first conducted with a specific refugee group and demonstrated the need for first aiders to have specific knowledge that can be categorised in four broad areas; (1) knowledge of the Iraqi culture and community in Australia; (2) how to communicate effectively with individuals from an Iraqi background (3) traditional beliefs about causes and negative attitudes towards mental health problems held by this group; and (4) common barriers to seeking professional help that can be present in resettled Iraqi refugees. These guidelines are relevant and needed, especially given that Iraq is one of the top-source countries of refugee applications to Australia. These guidelines represent the first step on the road in order to improve professional help-seeking in Iraqi refugees with mental health problems.

\section{Additional files}

Additional file 1. Endorsed items by category.

Additional file 2. Rejected items by category.

\section{Abbreviations \\ MHFA: Mental Health First Aid; ALG: Australian Libraries Getaway; UNHCR: United Nations High Commissioner for Refugees.}

\section{Authors' contributions}

MGU, SSY and AFJ conceived and designed the study. YS contributed substantially to the conception and the methodology used. MGU assisted with data collection and data management. All authors contributed in the data analysis. All authors contributed with revisions. All authors read and approved the final manuscript.

\section{Author details}

${ }^{1}$ Centre for Health Research, School of Medicine, Western Sydney University, Sydney, Australia. ${ }^{2}$ Health Promotion Service, Multicultural Health, South Western Sydney Local Health District, Sydney, Australia. ${ }^{3}$ Centre for Mental Health, Melbourne School of Population and Global Health, University of Melbourne, Melbourne, Australia.

\section{Acknowledgements}

We would like to thank Dr. Kathy Bond from MHFA Australia for providing excellent methodological expertise to this study.

\section{Competing interests}

Professor Anthony Jorm is Chair of Board of Mental Health First Aid International (trading as Mental Health First Aid Australia). This is a not-for-profit company with charity status. Jorm is not paid for this role.

\section{Availability of data and materials}

The datasets supporting the conclusions of this article are included as part of the additional file section (Additional files 1, 2). It is important to note that the ratings from each participant cannot be shared in order to comply with the Western Sydney University Ethics policy.

\section{Ethics approval and consent to participate}

This research was granted human research ethics approval by the Western Sydney University Human Research Ethics Committee reference number H11054.

\section{Funding}

Funding for this research was provided via an Australian Postgraduate Award from Western Sydney University and a 2015 Cross-cultural Public Research grant awarded to Ms Maria Gabriela Uribe Guajardo.

Received: 14 June 2016 Accepted: 22 August 2016

Published online: 01 September 2016

\section{References}

1. Australian Bureau of Statistics. Media release-National: Australia's multicultural landscape is as diverse as ever following the release of 2011 census of population and housing data by the Australian Bureau of Statistics (ABS). 2011. http://abs.gov.au/websitedbs/censushome.nsf/home/ CO-59. Accessed 14 Mar 2011.

2. Refugee Council of Australia: resources. 2014. http://www.refugeecouncil. org.au/resources/statistics/australias-refugee-and-humanitarian-program. Accessed 20 Apr 2105.

3. Department of Immigration and Border Protection: Australia's offshore humanitarian program 2013-2014. In: Department of Immigration and Border Protection. 2014. https://www.immi.gov.au/pub-res/Documents/statistics/ australia-offshore-humanitarian-program-2013-14.pdf. Accessed 1 Apr 2015.

4. Department of Immigration and Border Protection: Australia's response to the Syrian and Iraqi humanitarian crisis. 2015. https://www.border.gov. au/Trav/Refu/response-syrian-humanitarian-crisis. Accessed 7 Mar 2016

5. Mollica R, Wyshak G, Lavelle J. The psychosocial impact of war trauma and torture on Southeast Asian refugees. Am J Psychiatry. 1987;144:1567-72.

6. Steel Z, Chey T, Silove D, Marnane C, Bryant R, Ommeren M. Association of torture and other potentially traumatic events with mental health outcomes among populations exposed to mass conflict and displacement: a systematic review and meta-analysis. JAMA. 2009. doi:10.1001/jama.1132.

7. Porter M, Haslam N. Predisplacement and postdisplacement factors associated with mental health of refugees and internally displaced persons. JAMA. 2005. doi:10.1001/jama.294.5.602.

8. Slewa-Younan S, Uribe MG, Heriseanu A, Hasan T. A systematic review of post-traumatic stress disorder and depression amongst Iraqi refugees located in western countries. J Immigr Minor Health. 2014. doi:10.1007/ s10903-014-0046-3.

9. Uribe Guajardo MG, Slewa-Younan S, Smith M, Eagar S, Stone G. Psychological distress is influenced by length of stay in resettled Iraqi refugees in Australia. Int J Ment Health Syst. 2016. doi:10.1186/s13033-016-0036-z.

10. Correa-Velez I, Sundararajan V, Brown K, Gifford SM. Hospital utilisation among people born in refugee-source countries: an analysis of hospital admissions, Victoria, 1998-2004. Med J Aust. 2007;186(Suppl 11):577.

11. Youssef J, Deane FP. Factors influencing mental-health help-seeking in Arabic-speaking communities in Sydney, Australia. Ment Health Relig Cult. 2006;9(Suppl 1):43-66.

12. Bolton P. Mental health in Iraq: issues and challenges. Lancet. 2013;381(Suppl 9870):879-81.

13. Slewa-Younan S, Mond J, Bussion E, Mohammad Y, Uribe Guajardo MG, Smith M, et al. Mental health literacy of resettled Iraqi refugees in Australia: knowledge about posttraumatic stress disorder and beliefs about helpfulness of interventions. BMC Psychiatry. 2014. doi:10.1186/ s12888-014-0320-X.

14. Murray K, Davidson G, Schweitzer R. Psychological wellbeing of refugees resettling in Australia. In: The Australian Psychological Society. 2008. https://www.psychology.org.au/assets/files/refugee-lit-review.pdf. Accessed 4 Aug 2015 
15. Kitchener B, Jorm A. Mental health first aid training for the public: evaluation of effects on knowledge, attitudes and helping behaviour. BMC Psychiatry. 2000;2(Suppl 1):10.

16. Hadlaczky G, Hökby S, Mkrtchian A, Carli V, Wasserman D. Mental health first aid is an effective public health intervention for improving knowledge, attitudes, and behaviour: a meta-analysis. Int Rev Psychiatry. 2014. doi:10.3109/09540261.2014.924910.

17. Kitchener BA, Jorm AF. Mental health first aid: an international programme for early intervention. Early Interv Psychiatry. 2008. doi:10.1111/j.1751-7893.2007.00056.x.

18. Mendenhall AN, Jackson SC, Hase S. Mental health first aid USA in a rural community: perceived impact on knowledge, attitudes and behavior. Soc Work Ment Health. 2013. doi:10.1080/15332985.2013.812542.

19. Svennson B, Hansson L. Effectiveness of mental health first aid training in Sweden. A randomized controlled trial with a six-month and two-year follow-up. PLoS One. 2014. doi:10.1371/journal.pone.0100911.

20. Jensen KB, Morthorst BR, Vendsborg PB, Hjortoj C, Nordentoft M. Effectiveness of MHFA training in Denmark: a randomized trial in waitlist design. Soc Psychiatry Psychiatr Epidemiol. 2016. doi:10.1007/ s00127-016-1176-9.

21. Kitchener B, Jorm A, Kelly C. Mental Health First Aid International manual. Melbourne: Mental Health First Aid International; 2015.

22. Jorm AF. Using the Delphi expert consensus method in mental health research. Aust N Z J Psychiatry. 2015. doi:10.1177/000486741560089.

23. Hart L, Jorm AF, Kanowski L, Kelly C, Langlands R. Mental health first aid for Indigenous Australians: using Delphi consensus studies to develop guidelines for culturally appropriate responses to mental health problems. BMC Psychiatry. 2009. doi:10.1186/1471-244X-9-47.

24. Chalmers K, Bond K, Jorm AF, Kelly C, Kitchener B, Williams-Tchen A. Providing culturally appropriate mental health first aid to an Aboriginal or Torres Strait Islander adolescent: development of expert consensus guidelines. Int J Ment Health Syst. 2014. doi:10.1186/1752-4458-8-6.

25. Minas $\mathrm{H}$, Jorm AF. Where there is no evidence: use of expert consensus methods to fill the evidence gap in low-income countries and cultural minorities. Int J Ment Health Syst. 2010. doi:10.1186/1752-4458-4-33.

26. Summerfield D. A critique of seven assumptions behind psychological trauma programmes in war-affected areas. Soc Sci Med. 1999. doi:10.1016/S0277-9536(98)00450-X

27. Minas H, Kakuma R, San Too L, Vayani H, Orapeleng S, Prasad-IIdes R, et al. Mental health research and evaluation in multicultural Australia: developing a culture of inclusion. Int J Ment Health Syst. 2013. doi:10.1186/1752-4458-7-23.

28. May S, Rapee RM, Coello M, Momartin S, Aroche J. Mental health literacy among refugee communities: differences between the Australian lay public and the Iraqi and Sudanese refugee communities. Soc Psychiatry Psychiatr Epidemiol. 2014. doi:10.1007/s00127-013-0793-9.

29. Yaser A, Slewa-Younan S, Smith C, Olsen R, Uribe Guajardo MG, Mond J. Beliefs and knowledge about post-traumatic stress disorder amongst resettled Afghan refugees in Australia. Int J Ment Health Syst. 2016. doi:10.1186/s13033-016-0065-7.

30. Reavley N, Jorm AF: National survey of mental health literacy and stigma. In: Department of Health and Ageing. 2011. http://pmhg.unimelb.edu. au/research_settings/general_community/?a=636496. Accessed 20 May 2016.

31. Reavy K, Hobbs J, Hereford M, Crosby K. A new clinic model for refugee health care: adaptation of cultural safety. Rural Remote Health. 2012;12:1826.
32. Uitterhaegen B. Psycho-education and psychosocial support in the Netherlands: a program by and for refugees. Intervention. 2005;3(Suppl 2):141-7.

33. Hart LM, Bourchier SJ, Jorm AF, Kanowski LG, Kingston AH, Stanley D, Lubman DI. Development of mental health first aid guidelines for Aboriginal and Torres Strait Islander people experiencing problems with substance use: a Delphi study. BMC Psychiatry. 2010. doi:10.1186/1471-244X-10-78.

34. Steel Z, Chey T, Silove D, Marnane C, Bryant RA, van Ommeren M. Association of torture and other potentially traumatic events with mental health outcomes among populations exposed to mass conflict and displacement: a systematic review and meta-analysis. JAMA. 2009. doi:10.1001/ jama.2009.1132.

35. Sulaiman-Hill CM, Thompson SC. Thinking too much: psychological distress, sources of stress and coping strategies of resettled Afghan and Kurdish refugees. J Muslim Health. 2012;6(Suppl 2):63-86.

36. Jones J, Hunter D. Consensus methods for medical and health services research. BMJ. 1995;311(Suppl 7001):376-80.

37. Van De Ven A, Delbecq A. The effectiveness of nominal, Delphi, and interacting group decision making processes. Acad Manag J. 1974;17(Suppl 4):605-21.

38. Hardy DJ, O'Brien AP, Gaskin CJ, O'Brien Morrison-Ngatai E, Skews G, et al. Practical application of the Delphi technique in a bicultural mental health nursing study in New Zealand. J Adv Nurs. 2004. doi:10.1111/j.1365-2648.2003.02969.x.

39. Redmond M, Rooney R, Bishop B. Unipolar depression across cultures: a Delphi analysis of the methodological and conceptual issues confronting the cross-cultural study of depression. AeJAMH. 2006. doi:10.5172/ jamh.5.2.113.

40. Akins $\mathrm{R}$, Tolson $\mathrm{H}$, Cole B. Stability of response characteristics of a Delphi panel: application of bootstrap data expansion. BMC Med Res Methodol. 2005;5(Suppl 1):37.

41. Parcesepe A, Cabassa L. Public stigma of mental illness in the United States: a systematic literature review. Adm Policy Ment Health. 2013. doi:10.1007/s10488-012-0430-z

42. Chong S, Verma S, Vaingankar J, Huak Chan Y, Yin Wong L, Heng B. Perception of the public towards the mentally ill in developed Asian country. Soc Psychiatry Psychiatr Epidemiol. 2007;42(Suppl 9):734-9.

43. Iversen A, van Staden L, Hughes J, Greenberg N, Hotopf M, Rona $\mathrm{R}$, et al. The stigma of mental health problems and other barriers to care in the UK Armed Forces. BMC Health Serv Res. 2011. doi:10.1186/1472-6963-11-31.

44. Rickwood D, Thomas K, Bradford S. Review of help-seeking measures in mental health: an evidence check rapid review brokered by the Sax Institute for beyondblue. Sax Institute. 2012. https://www.saxinstitute.org. au/wp-content/uploads/02_Help-seeking-measures-in-mental-health. pdf. Accessed 7 Aug 2016

45. Fenta H, Hyman I, Noh S. Mental health service utilization by Ethiopian immigrants and refugees in Toronto. J Nerv Ment Dis. 2006. doi:10.1097/01.nmd.0000249109.71776.58

46. Sulaiman-Hill CM, Thompson SC. Selecting instruments for assessing psychological wellbeing in Afghan and Kurdish refugee groups. BMC Res Notes. 2010. doi:10.1186/1756-0500-3-237.

\section{Submit your next manuscript to BioMed Central and we will help you at every step:}

- We accept pre-submission inquiries

- Our selector tool helps you to find the most relevant journal

- We provide round the clock customer support

- Convenient online submission

- Thorough peer review

- Inclusion in PubMed and all major indexing services

- Maximum visibility for your research

Submit your manuscript at www.biomedcentral.com/submit 